\title{
El reino de Mari: ciudades, aldeas e identidades durante el período Paleobabilónico (2000-1600 a.C.)
}

\author{
(4) Leticia Rovira \\ Consejo de Investigaciones de la Universidad Nacional de Rosario / Universidad Nacional del \\ Litoral, Argentina
}

Fecha de recepción: 25 de julio de 2021. Fecha de aceptación: 18 de agosto de 2021.

\section{Resumen ${ }^{1}$}

En este breve artículo quisiéramos marcar unas líneas sobre dos tipos diferentes de asentamientos que se emplazaban en todo el reino de Mari, durante el período Paleobabilónico: las ciudades y las aldeas. A través del acercamiento a las fuentes del Archivo Real de Mari, notamos que para nombrar a los moradores del centro político de Mari se utilizan referencias más unidas a lo laboral que a otros diacríticos identitarios. En tanto en las urbes más pequeñas y aldeas las identidades que aparecen atestiguadas en las fuentes también ponen de relieve lo étnico, cuestión mayormente ocluida en la ciudad capital.

Palabras clave: Mari, período Paleobabilónico, ciudad, aldea, identidad

The Kingdom of Mari: Cities, Villages and Identities during the Old Babylonian Period (2000-1600 BCE)

\begin{abstract}
In this short contribution we would like elaborate some thoughts on two different types of settlements that were located throughout the kingdom of Mari, during the Old Babylonian period: cities and villages. In the Royal Archive of Mari, residents of the political centre of Mari are described using references closely linked to labour, rather than to other identity diacritics. In the smaller
\end{abstract}


cities and villages, however, the attested identities in the sources also highlight the ethnic issue, something that is mostly occluded in the capital city.

Keywords: Mari, Old Babylonian period, city, village, identity

Las ciudades son, por excelencia, un espacio socialmente construido (García de Cortázar, 1985: 7) por quienes las pueblan, lo que nos posibilita, en algunos casos, analizar esas sociedades en sus prácticas e identidades ligadas a tales urbes en tanto las alteran, las recorren, las manejan y las habitan entre otras acciones. Pero es importante no perder de vista que en el antiguo Cercano Oriente, las ciudades, cabeceras de reinos o más pequeñas, no se encontraban aisladas. Tales aglomeraciones fueron enclaves particulares en un mundo agrícola plagado de aldeas, las cual las sustentaban. En esta dirección la relación entre campo y ciudad es uno de los tópicos que encontramos en las fuentes paleobabilónicas de Mari. Además, en éstas se puede rastrear información sobre las identidades que se desenvolvían a partir de las pertenencias a diversas instalaciones campesinas y/o urbanas. Es entonces que nos interesa hacer algunas apreciaciones sobre dos formas de asentamiento que aparecen en el Archivo Real de Mari, las ciudades (älum), tomando como caso particular el emplazamiento de Mari como capital, para luego pasar a escudriñar urbes menores y aldeas (kaprum).

\section{La ciudad de Mari: esa intrigante (des)conocida}

En la zona denominada siro-mesopotámica encontramos el sitio arqueológico de Tell-Hariri, la importante antigua ciudad de Mari (älum mariki). Hoy en día ubicamos sus vestigios en Siria, a $12 \mathrm{~km}$ de la pequeña ciudad de Abu-Kamal y $15 \mathrm{~km}$ de la frontera con Iraq, situada a unos $21 / 2 \mathrm{~km}$ en la ribera derecha en la gran curva del Éufrates.

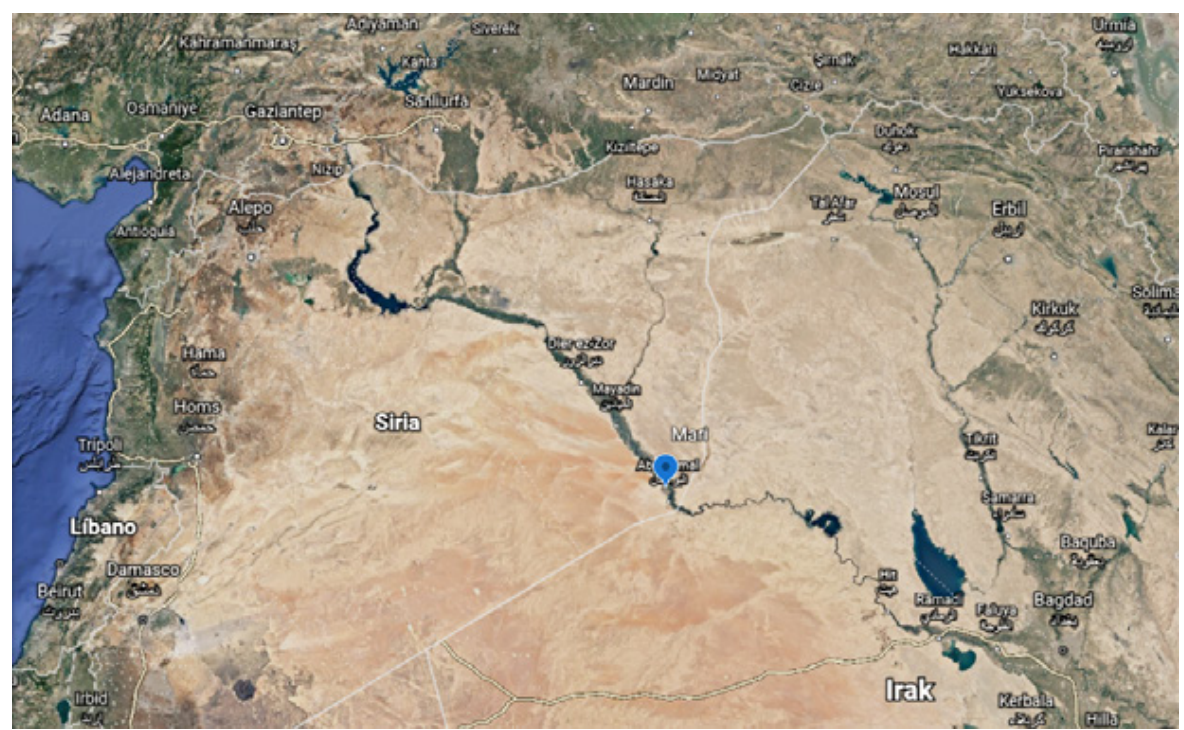


Este río, que fluye de noroeste a sureste, fue el mayor proveedor de agua de la ciudad durante toda su historia, utilizándose tanto para los cultivos como para la navegación. En el sitio de Tell Hariri-Mari los arqueólogos han hallado sobre una terraza holocena tres fases importantes de ocupación, ${ }^{2}$ las denominadas Ciudad I, II y III.

La Ciudad I (siglos XXX/XXIX ${ }^{3}$-XXVII a.C.) fue el momento de su fundación como eje de intercambio del Éufrates y sus afluentes, el Habur en primer lugar (Margueron, 2000: 103). El sitio fue abandonado al finalizar el Dinástico Arcaico II (ca. 2800-2600 a.C.) o principios del Dinástico Arcaico III (ca. 26002340 a.C.). A fines de ese período se refunda en lo que se da en llamar Ciudad II. Ésta sobrevivió entre los siglos XXVI y XXIII a.C. y hay rastros arqueológicos de una destrucción por la cual se plantearon varias hipótesis sobre quiénes la llevaron a cabo ya que es muy dificultoso poder trazar una cronología certera. ${ }^{4}$

En tanto la Ciudad III es la última fase de ocupación del sitio que comenzó durante el siglo XXIII a.C. y culminó en 1760 a.C. cuando fue finalmente quemada por el ejército de Hammurapi de Babilonia (1792-1750 a.C.). ${ }^{5}$ Ésta fue la ciudad ocupada más de 300 años por los Šakkanakku ${ }^{6}$ a fines del III milenio ${ }^{7}$ y luego por las dinastías amorreas en el II milenio. En 1760 a.C. finaliza la vida de la urbe que nunca se recuperó de ese infortunio. Su ocupación posterior en época medio-asiria y seléucida-parta, fue esporádica (Margueron, 2008: 35).

2 Síntesis de tales ocupaciones en Margueron, 2004; 2008.

3 Margueron (2008: 35) consigna que esta ocupación se dio alrededor del siglo XXX y el mismo autor (Margueron, 2000: 910), años antes planteaba el comienzo un siglo después, siglo XXIX.

4 Tal destrucción fue atribuida a Sargón (i. e. Charpin, 2008: 225, entre otros) o su nieto Narām-Sîn (Margueron, 2004: 310-311; 2008), pero un artículo de 2012 de Durand plantea que ésta se dio no como una conquista de esos reyes sino como un conflicto en Mari entre los gobernadores Šakkanakku (ver nota 6) que quisieron sacudirse el yugo del poder de Agade durante el reinado de Narām-Sîn (Durand, 2012: 131). En ese mismo artículo también explicita la hipótesis de que se podría pensar en una transición entre esa línea reinante y la amorrea que sería la que los destronaría dejando sin efecto el supuesto hiato que complicaba las cronologías entre estas dinastías (Durand, 2012: 131-132).

5 Diferentes hipótesis sobre la destrucción de Mari por Hammurapi: Charpin, 2004: 327330; Charpin y Ziegler, 2003: 261-262.

6 Este título que podría traducirse por "gobernador militar" fue dado a los mandatarios de Mari, por la dinastía fundada por Sargón de Agade y se mantuvo luego de la caída de ese poder regional. Tal titulación terminó representando la tradición propia del poder local, así como su legitimación (Durand, 1985: 158) por haber sido impuestos por un gran reino.

7 Cronología de los Šakkanakku, se puede ver en: Charpin, 2008: 227-228. En 2012 Durand revé algunas cuestiones al respecto (ver nota 4). 


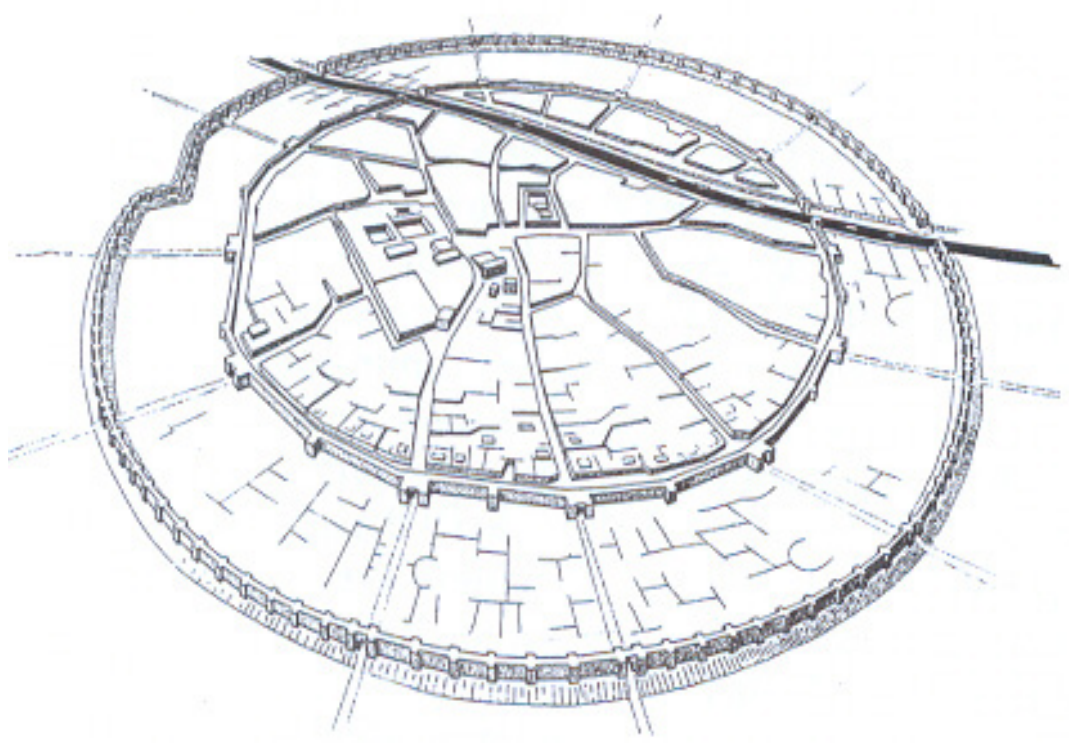

La Ciudad III (Margueron, 2004: 447).

La localización de la ciudad desde su nacimiento fue elegida de manera precisa. Indicio de ello es que se construyeron desde su mismo origen tres canales (Margueron, 2008): uno que derivaba el agua del Éufrates hacia la urbe, otro que servía para la irrigación de cosechas y huertos, y el tercero que se utilizaba para navegar, eligiéndolo antes que al río ya que disminuía y facilitaba el recorrido hacia la ciudad (Margueron, 2004: 72-79; 2008: 41-42, contra Durand, 2002b: 574-575). Es entonces que se entiende que para llevar adelante grandes proyectos de infraestructura como esos se necesitó seguramente un poder centralizado que pudiera organizar su desarrollo y a la vez abastecerse de mano de obra y también mantenerla para los trabajos. Además, se puede inferir que fue necesario un intercambio interregional así como terrenos agrícolas que nutrieran a los obreros que a la vez oficiaban de población de la emergente urbe (Margueron, 2008: 44).

Esta ciudad fue la capital del reino con su mismo nombre, el cual comprendía cuatro distritos (el de Mari, Terqa, Saggarātum y Qaț̣unān), la zona de control llamada Suhûm y las áreas de los gobiernos que subyugaba en el norte siromesopotámico. Toda esta zona de dominio directo e indirecto contaba con una diversidad de tipos de asentamientos, pero hay pocas estimaciones sobre el número de personas que la habitaban y se supone que equivalía a unas 50.000 personas (Durand, 2004: 111). En tanto en particular sobre la propia ciudad de Mari, su población no debía ser una gran cantidad y la mayoría de ella se distribuía en la campiña vecina (Durand, 1998: 520-521).

Una de las repetidas y sabidas nociones para distinguir un asentamiento como una ciudad de uno que no lo era tiene que ver con lo fuertemente estructuradas que estaban las urbes en el Cercano Oriente antiguo en cuanto a lo edilicio. En ellas encontramos en primera instancia los palacios y templos, como centros rectores del poder terrenal y divino. En la propia ciudad de Mari se da 
la particularidad de encontrar un recinto sagrado dentro del llamado "Gran palacio", aunque también se asentaban en ella otros templos y un "Pequeño palacio oriental" (Margueron, 2004: 446-451). Pero además de estos grandes e imponentes edificios para su época, también se podían ubicar en las ciudades viviendas y talleres artesanales.

Una característica clásica, aunque no por ello menos ambigua y discutida para poder definirlas, era la de contar con murallas (Frangipane, 2018: 15). A Mari la encontramos rodeada por dos de ellas (Margueron, 2004: 443-446; 2008: 49-61). Mari tenía forma circular con un diámetro total de $1,9 \mathrm{~km}$ rodeada por una muralla como la primera línea de defensa. En tanto el centro de la ciudad totalizaba en diámetro $1,3 \mathrm{~km}$ y estaba protegido por una segunda muralla de la cual hay evidencia desde la Ciudad I. Posteriormente a tal ocupación se sabe que este sistema defensivo fue rehabilitado, pero no se tienen certezas sobre si ello fue llevado adelante por los habitantes de la Ciudad II o la III. Se supone que entre una y otra de estas murallas, en lo que se da en llamar "la corona", se encontraban jardines y huertos.

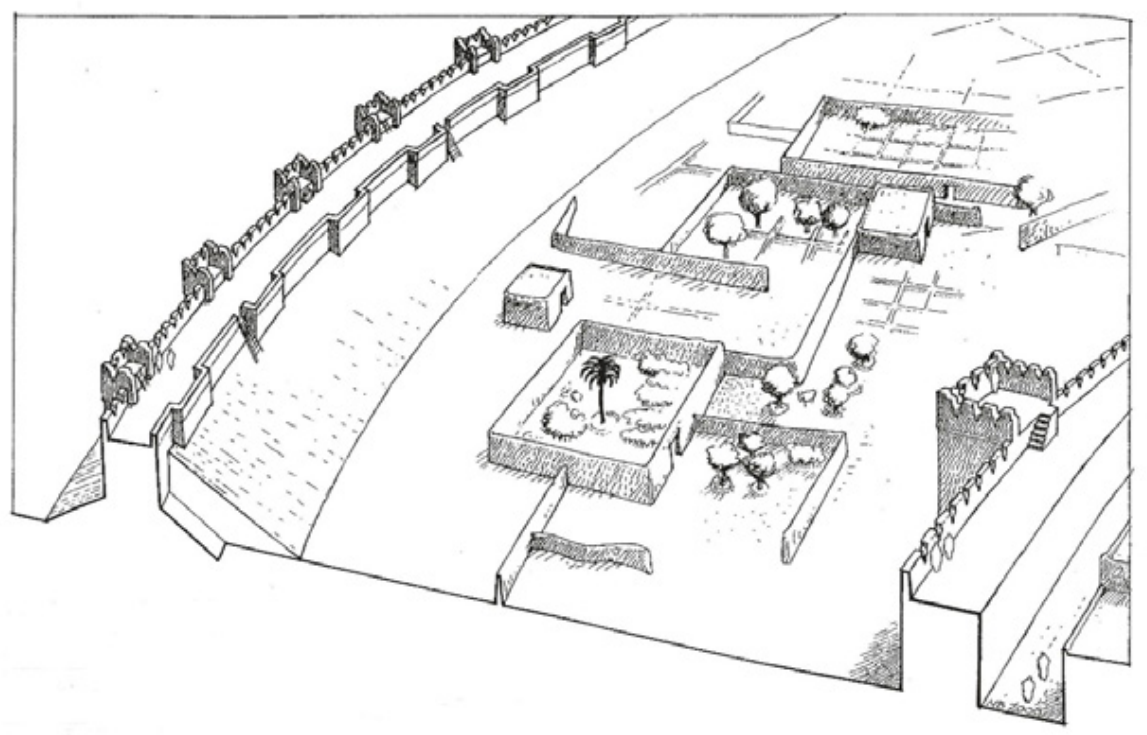

El sistema defensivo de doble muralla y entre ellas los jardines y huertos de la Ciudad III (Margueron, 2004: 446).

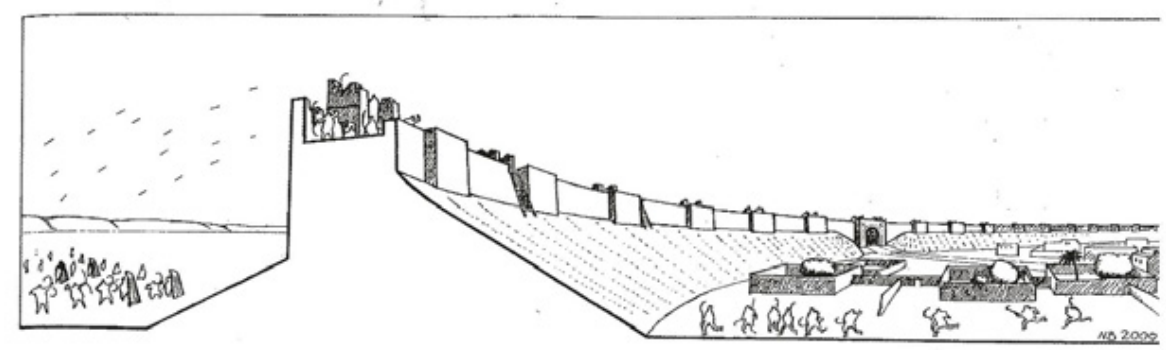

Muralla exterior en escala (Margueron, 2004: 447). 
Estas cuestiones estructurales de la urbe nos dan un marco para los retazos de información con los que contamos en lo que se refiere a las características internas y al funcionamiento de la ciudad de Mari en sí misma. En ella se registran diversos bābtum. Estos no tenían el sentido de "barrio/vecindario" como en Babilonia, sino que fueron unidades de trabajo, pero no divididos por un mismo oficio, y la gente que los conformaba era personal que residía en las aldeas vecinas o eran extranjeros (Durand, 1998: 520, Margueron, 2004: 443; Millet Albà, 2006: 305, 312). Cada uno de esos sectores era designado con el nombre de algún individuo que oficiaba de jefe o encargado del mismo, los llamados wakil bābātim, pero no sabemos cómo se los escogía ni si eran funcionarios ocasionales o permanentes del palacio (Durand, 1987: 664; Durand, 1997: 222; Millet Albà, 2006: 305).

Varios $b \bar{a} b t u m^{8}$ son nombrados en el documento ARM XXII 13, los cuales estaban bajo la tutela de diferentes individuos. En esta fuente se enumeran tales diversas secciones y además del apelativo de sus jefes, encontramos el de las personas que estaban bajo sus órdenes. Estos últimos son listados por sus nombres de pila, sin ninguna otra filiación, sólo como parte de uno de esos bābtum, por ejemplo:

(...)Tir-Mamma, Hilla, Qišti-Tabutu: Sección de Zabinum (ba-ab-ti Za-bi-nim) (...) (ARM XXII 13: 9'-12').

O de esa misma forma y a ello se sumaba un número particular de personas con algún oficio (carpinteros, orfebres, braseros, etc.), por ejemplo:

(...) 13 herreros, Sin-lšmeni, Ili-Haznāya, Qİs̆atum: Sección de Ana-Ea-Takläku (ba-ab-ti A-na $\left.{ }^{d} E-a-t a ́ k-l a-k u\right)(. .).(A R M X X I l$ 13: 17-21).

Estos grupos de trabajadores con algún oficio eran los que podían ser denominados trabajadores especializados (mārū ummênīm/ DUMU.MEŠ ummênīm) (Durand, 1997: 221; Zaccagnini, 1983: 247; Matthews, 2006 [1995]: 455; Rovira, 2011).

También encontramos en el entramado de la ciudad bajo la vigilancia del prefecto de los mercaderes (PA DAM.GÀR) a "la gente del puerto" (mārū kārum / DUMU.MEŠ KAR.TA). Estos pudieron haber sido extranjeros que vivían en la urbe y habitaban un espacio específico. ${ }^{9}$ Tal expresión se supone englobaba, según Durand (1987) ${ }^{10}$ a todos los individuos que realizaban actividades

8 Varias son las fuentes en donde se puede encontrar referencia a los bäbtum; las enumera Millet-Albà (2006) pero todavía se encuentran inéditas y no son parte de ARCHIBAB por el momento.

9 Millet-Albà (2006) y Durand (1987) comparan a estos individuos con los metecos atenienses.

10 Durand (1997: 222), hace referencia a este grupo, recurriendo a una imagen moderna, según sus dichos como "industriales" y los relaciona con una especie de "maestro herrero", sabiendo que éstos podían incluirse dentro del grupo de los mārū ummêním (ver Rovira, 2011). A su vez, Durand (1987), los compara con los metecos ateniense. No 
portuarias tales como barqueros, estibadores, comerciantes, etc. (Kupper, 1985: 464; Durand, 1987; Birot, 1993: 254) sin darnos demasiadas precisiones, y en la carta ARM XXVII 151 se los nombra:

(...) Cuando [ha inspeccionado]? a la gente del puerto (șa-ba-am KAR-TA gibil) -nuevos reclutas y veteranos, él (Ibâl-pî-El ${ }^{11}$ ) ha dado instrucciones a Šarrum-andulli (...) (ARM XXVII 151: 42-43).

Pocas son las menciones que se tienen sobre estos actores, una de ellas la encontramos en una fuente que no tenía como objetivo expresarse sobre algún tema relativo a trabajos o comercio sino informar sobre un suceso criminal por el cual se necesitaba información, y se apeló entonces a indagar a las personas que recorrían la ciudad con asiduidad. Esta fuente dice:

\section{(...) El mismo día que me enteré de esta historia, di las órdenes oportunas y presioné con preguntas a los jefes de sección, trabajadores especializados y gente del puerto ([L]Ú* UGULA ba-ba-tim DUMU.MEŠ um-me-ni [ù DU]MU.MEŠ KAR.TA*) (...) (ARM VI 43:16-19). ${ }^{12}$}

De los ejemplos de fuentes que hemos tomado en este desarrollo quisiéramos retener el término DUMU/mārum "hijo", en sumerio y acadio respectivamente. La aparición de DUMU/mārum en diversas instancias de definiciones identitarias nos subraya la sabida importancia capital de las relaciones parentales en tanto forjadoras de lazos. Su utilización denotaba identidades sociales que correspondían tanto a labores como a clanes, tribus ${ }^{13}$ y etnias ${ }^{14}$; la asociación a un determinado espacio geográfico y, a su vez, la marca de subordinación de una persona a otra.

Es entonces que nos interesa la utilización de DUMU que se encuentra en las cartas cuando marcan una relación con lo territorial indicando a las personas de diversas ciudades. En esta dirección DUMU.MEŠ à lim $^{\mathrm{ki}}$ "hijos de la ciudad" fue una de las formas de llamar a los citadinos: ${ }^{15}$

(...) Que los citadinos (DUMU.MEŠ $a$-limi) sean sacados del recinto fortificado y que vivan en las afueras para que la tropa de guarnición mantenga fuertemente el recinto

queda muy claro cuál es su idea general. Retengamos que podían ser extranjeros domiciliados en Mari que se relacionaban con el puerto.

11 Enviado de Mari a Babilonia.

$12=$ LAPO 181062.

13 Se debe tener presente que la etnia bensimalita se dividía en clanes (gayum) y la etnia benjaminita en tribus (lïmum) (entre otros ver Durand, 2004; Fleming, 2004).

14 Para la definición de etnia ver De Bernardi, 2005.

15 En ARM XXXIII, según Durand (p. 465 n.a contra CAD A/1: 334) alānûm significa "citadino que se encuentra lejos de su casa", alguien que puede estar llevando adelante rutas de trashumancia, pero no como un "verdadero transhumante", el cual sería hanûm. Ver la fuente ARM XXXIII 224. Con alānûm entonces tendríamos otra forma de saber quiénes pertenecían a un asentamiento. 
fortificado. Además, se debe poner atención en no permitir que ningún citadino (DUMU. MEŠ $\left.a-l i{ }^{k i}\right)$ entre en el recinto fortificado. ${ }^{16}(. .).($ A.315+: 25-31).

Pero para la ciudad de Mari es difícil encontrar una gran cantidad de fuentes en donde se nombre a sus moradores como meros residentes en ella. Tres cartas únicas nos marcan formas diferentes y no habituales de enunciar a los habitantes de la capital del reino.

En primer término, como DUMU, que es la forma más habitual en cuanto a referencia geográfica, pero no así para la capital mariota:

$$
\text { (...) } 1 \text { persona de Mari (1 LÚ ša DUMU.MEŠ॰ }\left\ulcorner\text { ma ?-ri }{ }^{k i}\right)(. . .)(F M 723: 9) .{ }^{17}
$$

Por otro lado, LÚ (persona) pero sin resaltarse como "perteneciente a" apelando a nombrarlo como "hijo"

(...) las personas de Mari ( $($ LÚ 7.MEŠ ša i-na ma-riki ) (...) (A. 1333: 24’).

Y una tercera forma que retoma la noción de citadino a partir de DUMU/ mārum pero a su vez se remarca su identidad de una ciudad particular, Mari, ya que son "hombres de Mari", residentes de esa urbe, pudiendo entender esa denominación de "hombres" como "personas" en tanto la población que ha sido echada incluiría a hombres, mujeres y niños.

(...) Desde que habías hecho salir a los citadinos de Mari, personas de esa ciudad

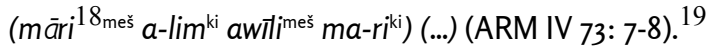

Dos de estas fuentes (A.1333 y ARM IV 73) pertenecen al período de reinado de la Alta Mesopotamia. La tercera (FM 7 23) al de Zimrī-Lîm que es también el período al que pertenece ARM XXII 13 en donde algunas de las personas que pudieron llegar a habitar la ciudad capital eran nombradas a partir de identificarlas por sus nombres de pila o en relación a un oficio, por estar bajo

16 (25) DUMU.MEŠ a-lim iš-tu ki-ir-hi-im li-še-șú-ma (26) i-na a-da-aš-ši-im ${ }^{k i}$ li-iš-bu (27) ù șa-bu-um bi-ir-tum i-na e-mu-qi-im (28) ki-ir-ha-am li-ki-il (29) ù nu-uh-hi-id-ma i-na DUMU.MEŠ a-lim ki (30) 1 LÚ a-na ki-ir-hi-im a-na e-re-bi-im (31) la i-na-ad-di-un. Una de las acepciones de kirhum, la que cuenta en este caso, es la de muralla interior de una ciudad, la que protege el corazón de la misma. En tanto adaššum sería la muralla exterior de una urbe. Se entiende entonces que esta carta hace alusión a que vivan fuera del recinto fortificado (el kirhum) y sería que se emplacen por fuera del adaššum. Ver al respecto Dossin, 1972 y Margueron, 2004: 446.

17 A diferencia de la línea citada, la siguiente, la 10, dice 1 LÚ ga-n[i]-ba-tim ${ }^{[k]}$, "1 persona de Ganibatum", en ésta no encontramos el DUMU que es el que daría pertenencia a ese espacio geográfico. Esto se puede deber a que Ganibatum era el puerto de la ciudad de Dūr-Yahdun-Lîm. En las cartas de Mari otros ejemplos de DUMU como población de una ciudad determinada se pueden ver en varios casos por ejemplo en ARM I 75, en relación a Ekallātum y en ARM III 3 en relación a Terqa.

18 māri = DUMU. 
la supervisión de una persona en particular relacionada a esos trabajos en tanto jefes de los bābtum o por ser extranjeros afines a cuestiones comerciales, pero no se los nombraba como población general de la ciudad.

Es entonces que esas designaciones y las pocas menciones que tenemos de los pobladores de la ciudad como tales hace que se pueda pensar que los citadinos de Mari eran mayormente identificados no por su pertenencia e identidad sujeta a un domicilio. Esto se podía deber a que en realidad sus vidas particulares transcurrían por fuera de ella en la campiña vecina y se movilizaban hacia la ciudad capital sólo a trabajar, una típica relación campo-ciudad. En cuanto a los que eran extranjeros, relacionados con el puerto, y que sí podían residir en la ciudad, eran dependientes del palacio a través de raciones (Millet-Albà, 2006: 312). Es entonces que, tanto unos como otros rendían servicio y ello impactaba en la forma de definirlos desde el estado que lo hacía identificándolos a partir de sus relaciones laborales, lo cual quedaba plasmado mayoritariamente en las fuentes con las que contamos. En esta dirección la ciudad misma tuvo una fisonomía y organización ligada a la explotación de la fuerza de trabajo, especializada o no. La identidad laboral, agrupada bajo la égida de un "jefe", fue mucho más destacada y operativa que la de mero habitante o extranjero en la ciudad o que la identidad étnica, ${ }^{20}$ a la hora de tener que ser registrados los sujetos por las autoridades.

\section{...y demás asentamientos...identidades en otros conglomerados urbanos y aldeas}

Como dijimos el reino mariota contaba también con ciudades (ālum) menores en relación a la capital, así como con aldeas (kaprum). Los benjaminitas y bensimalitas, las dos etnias más importantes del reino (ver Rovira, 2019), aunque no las únicas, residían en ambos tipos de conglomerados además de otros sitios. ${ }^{21}$

No puede atribuirse una sola forma de asentamiento particular según la etnia de pertenencia, pero se puede rastrear una ocupación geográfica diferencial o mayoritaria de territorios que se relacionaban con ellas. ${ }^{22}$

20 La onomástica referida en las fuentes en relación con la ciudad de Mari nos plantea nombres amorreos y acadios en algunos casos pertenecientes a una misma familia o a un mismo bābtum (Kupper, 1983a). Por lo cual no podemos utilizar esos datos para hacernos un cuadro de situación étnica de la ciudad y es probable que el dominio de las diferentes dinastías ligadas a la etnia benjaminita, los reyes de la Alta Mesopotamia, y a la bensimalita, los Lîm, hiciera también irrelevante marcar estas identidades en lo referente a la ciudad capital que dominaban. Aun así, encontramos fuentes en donde se explicitan tales diferencias en relación al poder de dominación y la pertenencia étnica, por ejemplo en: ARM VI 76, ARM XXVI/1 5, A.3274, entre otras.

21 Por ejemplo, los campamentos - mahanum- y tiendas -(h)urpatum- o las ciudades fortalezas como los dūrum, que no serán tratados aquí.

22 Se consignas las etnias mayoritarias en cada sitio, cuando se tiene la información, en: Ziegler y Langlois, 2016. 
Los benjaminitas, ocupaban especialmente el noroeste del reino desde Samânum, a Tuttul, todo el Zalmaqum, llegando a Yamhad - Alepo, hasta Ugarit en la costa del Mediterráneo y Qațna sobre el río Orontes. En tanto la implantación geográfica de los bensimalitas iba desde la ciudad de Appān, río arriba de Mari, llegando hasta Hiit como última localidad del Suhûm (Durand, 2004: 155-157), y también en el Ida-Maraș (Reculeau, 2008: 346).

Los benjaminitas poseían ciertas ciudades que eran denominadas como ālum. Algunas como Mišlân (Anbar, 1985) y Samânum tuvieron murallas, las cuales fueron desmanteladas por Zimrî-Lîm (Durand, 1998: 516-517). Una carta de Bannum a ese gobernante, que debe ser datada al principio de su reinado nos habla de tales asentamientos:

(...) Todos los benjaminitas han levantado la antorcha: desde Samânum hasta llummuluk, de llum-muluk hasta Mišlân. Todas las ciudades de los benjaminitas (a-la-nu ka-lu-šu-nu ša DUMU ia-mi-na- $a^{\circ}$ ) del distrito de Terqa han levantado la antorcha en respuesta.(...) (A. 56: 9-16). ${ }^{23}$

Como planteamos más arriba la identidad de DUMU.MEŠ àlim" "hijos de la ciudad", presentando a los citadinos, podía complementarse con otras adscripciones identitarias referidas a trabajos o la filiación a una etnia, tribu o clan. Un ejemplo es esta carta, A.56, encontrando en ella que la referencia a un asentamiento era reforzada por alguna de estas relaciones, en este caso particular la pertenencia étnica, ya que se le atribuyen ciudades a los benjaminitas. Esto reafirma que DUMU no sólo remite a una cuestión de identidades territoriales sino también a identidades étnicas y laborales, como vimos en el apartado anterior:

“(...) Por cierto decimos: «Tal ciudad es bensimalita pero tal es benjaminita» (a-lum annu-um DUMU si-im-a-al ù a-lum an-nu-um DUMU ia-mi-na) (...)" (A.3080: 19-21). ${ }^{24}$

A su vez, una y otra etnia aparecen agrupadas en kaprum. ${ }^{25}$ Esta palabra definía a los pequeños conglomerados de gente asentada en el campo abierto, granjas, sitios suburbanos alrededor de una ciudad, lo que se podría definir como "aldea". ${ }^{26}$ Tales kaprum eran una realidad menor a las ciudades, pero mayor a las simples y móviles tiendas (hurpatum), y podrían haber sido casas habitación rodeadas por vallas (Durand, 1998: 519).

23 LAPO 17683 = ARM XXXIII 36.

24 LAPO 17733.

25 Durand 1998: 519. CAD K: 189-190, 2'.

26 Ver Brown et al., 1996: 499; Agmon y Bloch, 2013: Table S3. Es interesante que el término kaprum puede rastrearse hasta hoy en día en el uso corriente en el árabe palestino كُمر (kafr) y en el hebreo moderno כopr) para definir asentamientos aldeanos (Milevski, 2010: 166). 
(...) Toda la población así como el grano de las aldeas de los bensimalitas (ša kapra-tim ša DUMU-MEŠ si-im-a-al) ha sido reunido en las plazas fuertes ${ }^{27}$ (...) (ARM XIV 84: $27-28) .{ }^{28}$

Di a Zimrī-Lîm, mi padre: así habla tu hijo, Dādī-hadun. ${ }^{29}$

Todas las diferentes ciudades (a-la-nu-ú) pertenecen a mi padre y nosotros mismos somos tus hijos. Sin embargo, tú sabes que en este país, mientras que mis hermanos recibieron la tenencia de varias aldeas ( $k a$-ap-ra-tim), yo he sido perjudicado: dos de mis aldeas (2 ka-[a]p-ru-ia) han sido tomadas. (...) (ARM II 61: 1-11). ${ }^{30}$

Para la ciudad de Mari, centro del poder del reino, se nos oscurece el acercamiento a las identidades étnicas, tribales o clánicas en relación a sus moradores en tanto como vemos podemos recoger mayor información sobre ellas en las urbes más pequeñas o aldeas aledañas.

Se puede pensar que la homogeneidad de los asentamientos que no eran la capital hacía más fácil el poder identificar ciertas identidades étnicas o que las mismas eran más en esos contextos en cuanto a sus relaciones con otras comunidades. Pero a su vez no se puede soslayar que ello está relacionado con una cuestión clasificatoria que servía al poder central (del cual emanaron la mayoría de las fuentes con las que contamos) como forma de control de las colectividades a partir de conocer determinadas realidades y con esa información estar al tanto de cómo manejarlas. ${ }^{31}$

\section{Algunas conclusiones}

En este trabajo intentamos exponer una arista de cómo eran nombradas diversas identidades sociales desde el aparato estatal del reino de Mari, para clasificar personas y lugares. Partimos de indagar y caracterizar a la ciudad capital, Mari, como un caso especial para luego acercarnos a la división entre ciudades, en ocasiones menores, y aldeas en tanto formas diferenciadas de asentamiento. En éstos, las identidades que se entrevén en las fuentes con más fuerza se encuentra relacionadas con lo étnico, en contraste con la ciudad de Mari en donde hallamos a sus habitantes (?) ligados a identidades sobre todo laborales. En esta dirección tales urbes y aldeas muchas veces eran a su vez referenciadas por sus adscripciones étnicas, tribales y clánicas. Esto no quiere decir que todos

27 dan-na-tim: lugares indefinidos que debían servir de protección de alguna forma que no podemos determinar con exactitud.

28 LAPO 17700 y en Charpin y Durand, 1986: 176-178.

29 Rey benjaminita de la tribu de los rabbûm y hermano de Addu-dūrī, madre de ZimrīLîm (Durand, 2000: 273-275; 2004: 185).

30 LAPO 17703.

31 Ejemplo de ello es la carta de ARM I 6= LAPO 17 641, que Samsī-Addu le envía a su hijo Yasmah-Addu en donde le aconseja no censar a los benjaminitas para que no se produzca un malestar innecesario en esa comunidad. 
los asentamientos por fuera de la capital fueran homogéneos en su geografía humana, sino que nos da indicios de que el poder palacial pudo llegar a tener una estrategia clasificatoria que le era necesaria para manejar los vínculos con tales comunidades o para dejar registrados determinados procedimientos relacionados con los diversos grupos identitarios que los habitaban. En esta dirección se marca un contraste con Mari como ciudad capital en donde las referencias laborales y la dependencia que tenían sus habitantes (?) con respecto al centro de poder palacial pudo ser la manera más clara para denominar a los grupos que se instalaban/transitaban por ella.

\section{Abreviaturas}

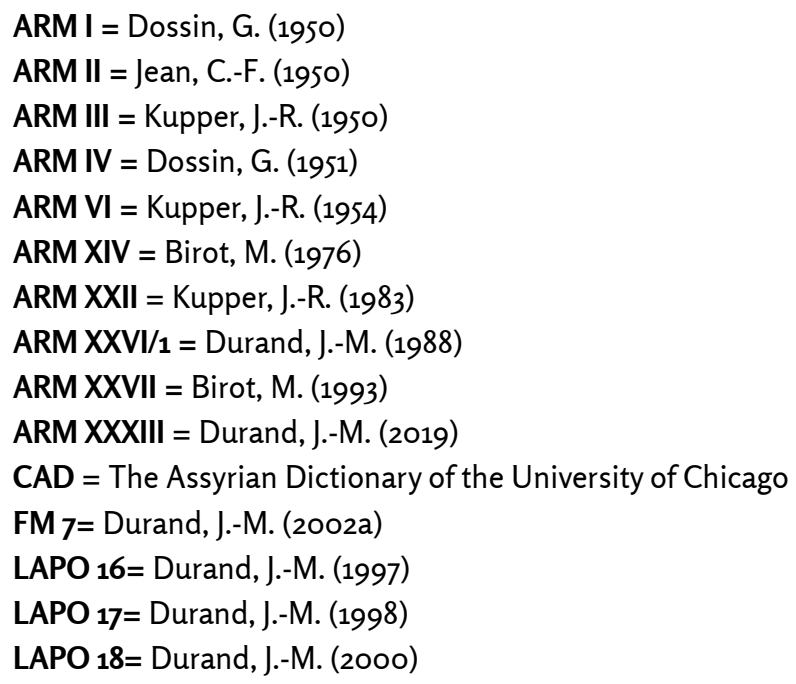




\section{Q Bibliografía}

»Agmon, N. y Bloch, Y. (2013). Statistics of Language Morphology Change: From Biconsonantal Hunters to Triconsonantal Farmers, en: PLoS ONE 8(12): e8378o. https://doi.org/10.1371/journal.pone.0083780. Table S3: https://doi.org/10.1371/ journal.pone.0083780.5004

"Anbar, M. (1985). La distribution géographique des Bini-Yamina d'après les archives royales de Mari, en: Birot, M., Durand, J.-M. y Kupper, J.-R., Miscellanea Babylonica. Mélanges offerts à Maurice Birot. París: Éditions Recherche sur les Civilisations, 17-24.

»Birot, M. (1976). Lettres de Yaqquim-Addu de Sagarâtum, ARM XIV. París: Paul Geuthner.

"Birot, M. (1993). Correspondance des gouvemeurs de Qatțunân, ARM XXVII. París: Éditions Recherche sur les Civilisations.

»Brown, F., Driver, S. y Briggs, C. (1996). The Brown-Driver-Briggs Hebrew and English Lexicon. Peadboy: Henrickson Publishers.

"Charpin, D. (2004). Histoire politique du Proche-Orient amorrite (2002-1595), en: Charpin, D., Edzard, D. O. y Stol, M., Mesopotamien. Die altbabylonische Zeit. OBO 160/4. Friburgo-Gotinga: Academic Press-Vandenhoeck \& Ruprech, 25-480.

»Charpin, D. (2008). Mari au Ille millénaire d'àpres les sources écrites, Tell Hariri/ Mari: Textes, en: Supplément au dictionnaires de la Bible 14: 77-78, col. 221-233.

»Charpin, D. y Durand, J.-M. (1986). 'Fils de Sim'al': Les origines tribales des rois de Mari, en: Revue d'Assyriologie et d'Archéologie Orientale 8o (2): 141-183.

"Charpin, D. y Ziegler, N. (2003). Florilegium Marianum V. Mari et le proche-Orient a l'epoque amorrite. Essai d'histoire politique, Mémoires de N.A.B.U. 6. París: SEPOA.

»De Bernardi, C. (2005). Methodological Problems in the Approach to Ethnicity in Ancient Mesopotamia, en: Van Soldt, W. H. (ed.), Ethnicity in Ancient Mesopotamia. Papers Read at the 48th Rencontre Assyriologique International, Leiden, 1-4 July 2002. Lovaina: Nederlands Instituut Voor Het Nabije OostenPeeters, 78-89.

»Dossin, G. (1950). Correspondance de Šamši-Addu et de ses fils, ARM I. París: Imprimerie Nationale.

"Dossin, G. (1951). Correspondance de Šamši-Addu, ARM IV. París: Imprimerie Nationale.

"Dossin, G. (1972). Adaššum et kirhuum dans des textes de Mari, en: Revue d'Assyriologie et d'archèologie orientale 66 (2): 111-130.

»Durand, J.-M. (1985). La situation historique des Šakkanakku: Nouvelle approche, en: Mari Annales de Recherches Interdisciplinaires 4: 147-172.

»Durand, J.-M. (1987). La population de Mari, en: Mari Annales de Recherches Interdisciplinaires 5: 664-665.

»Durand, J.-M. (1988). Archives Epistolaires de Mari, ARM XXVI/1. París: Éditions Recherche sur les Civilisations. 
»Durand, J.-M. (1997). Documents épistolaires du Palais de Mari, Vol. I, Littératures Anciennes du Proche-Orient 16 (LAPO 16). París: Les éditions du Cerf.

»Durand, J.-M. (1998). Documents épistolaires du Palais de Mari, Vol. II, Littératures Anciennes du Proche-Orient 17 (LAPO 17). París: Les éditions du Cerf.

»Durand,J.-M. (2000). Documents épistolaires du Palais de Mari, Vol. III, Littératures Anciennes du Proche-Orient 18 (LAPO 18). París: Les éditions du Cerf.

"Durand, J.-M. (2002a). Florilegium Marianum VII. Le culte du dieu de l'orage d'Alep et l'affaire d'Alahtum, Mémoires de N.A.B.U. 6. París: SEPOA.

»Durand, J.-M. (2002b). La maîtrise de l'eau dans les régions centrales du ProcheOrient, en: Annales, Histoire, Sciences sociales 57(3): 561-576.

»Durand, J.-M. (2004). Peuplement et société à l'époque amorrite (I) Les clans bensim'alites, en: Nicolle, C. (Ed.) Nomades et sedentaires dans le Proche-Orient ancien. Compte rendu de la XLVIe Rencontre Assyriologique Internationale, Amurru 3. París: Éditions Recherche sur les Civilisations, 111-197.

»Durand,J.-M. (2012). Sargon a-t-il détruit la ville de Mari?, en: Revue d'Assyriologie et d'archéologie Orientale 106(1): 117-132.

"Durand, J.-M. (2019). Les premières années du roi Zimrî-Lîm de Mari : Première partie, ARM XXXIII. Lovaina-París-Bristol: Peeters.

»Fleming, D. (2004). The sim'alite gayum and the yaminite li'mun in the Mari Archives, en: Nicolle, C. (Ed.) Nomades et Sédentaries dans le Proche-Orient Ancien. Compte rendu de la XLVIe Rencontre Assyriologique Internationale. Amurru 3. París: Éditions Recherche sur les Civilisations, 199-212.

» Frangipane, M. (2018) Introduction. The Many Dimension of the "City" in Early Societies, en: Origini 42: 13-23.

» García de Cortázar, J. A. (1985). La organización del espacio en la España medieval. La corona de Castilla en los siglos VII al XV. Barcelona: Ariel.

» Jean, C.-F. (1950). Lettres diverses, ARM II. París: Imprimerie Nationale.

» Kupper, J.-R. (1950). Correspondance de Kibri-Dagan, ARM III. París: Imprimerie Nationale.

» Kupper, J.-R. (1954). Correspondence de Bahdi-Lim, Prefet du Palais de Mari, ARM VI. París: Imprimerie Nationale.

»Kupper, J.-R. (1983a). Mari, en: La ville dans le Proche-Orient ancien. Lovaina: Peeters, 113-121.

»Kupper, J.-R. (1983b). Documents administratifs de la salle 135 du Palais de Mari, ARM XXII. París: Éditions Recherche sur les Civilisations.

» Kupper, J. R. (1985). La cité et le royaume de Mari. La organization urbaine à la époque amorrite, en: Mari Annales de Recherches Interdisciplinaires 4: 663-666.

»Margueron, J.-C. (2000). Mari et le Khabur, en: Rouault, O. y Wäfle, M. (Eds.) La Djéziré Et l' Euphrate Syriens. De La Protohistoire á la Fin du lle Millénaire Av. J.-C., Tendances Dans L'Interprétation Historique Des Données Nouvelles (Subartu VII). Turnhout: Brepols, 99-110.

" Margueron, J.-C. (2004). Mari: Métropole de l'Euphrate, au Ille et au début du lle millénaire av. JC. París: Picard-ERC.

" Margueron, J.-C. (2008). Tell Hariri/Mari. Archéologie, en: Supplément au Dictionnaire de la Bible 77-78: 17-214. 
» Matthews, D. (2006 [1995]). Artisans and Artists in Ancient Western Asia, en: Sasson, J. (ed. in chief), Civilization of the Ancient Near East, Vol. I-II. Nueva York: Hendrickson, 455-468

» Milevski, I. (2010). Centros urbanos y periferias en la Edad del Bronce Antiguo sud-levantina, en: Rivista degli Studi Orientali Nova Serie 83 (1-4): 163-187.

" Millet Albà, A. (2006). Le bābtum à Mari, en: Del Olmo Lete, G., Feliu, L. y Millet Albà, A. (Eds.) Shapal tibnim mû illaku. Studies presented to Joaquín Sanmartín on the Occasion of His 65th Birthday. Barcelona: AUSA, 303-313.

» Reculeau, H. (2008). Les sédentaires, en: Supplément au Dictionnaire de la Bible 14 (fasc. 77-78): col. 324-356.

"Rovira, L. (2011). Posibilidades y límites de la aplicación del concepto marxista de división del trabajo en la sociedad mariota (Siria - siglo XVIII a.C.), en: Ames, C. y Sagristani, M. (comps.), Actas de las III Jornadas Nacionales de Historia Antigua - II Jornadas Internacionales de Historia Antigua. Córdoba: Universidad Nacional de Córdoba, 56-72.

» Rovira, L. (2019). Ethnic and Pan-Ethnic Identity in The Kingdom of Mari (18th Century BC). A Model Kit, en: Oriens Antiqvvs. Serie Nova 1: 149-156.

»Zaccagnini, C. (1983). Patterns of Mobility among Ancient Near Eastern Craftsmen, en: Journal of Near Eastern Studies 42 (4): 245-264.

»Ziegler, N. y Langlois, A.-I. (2017). Les toponymes paléo-babyloniens de la HauteMésopotamie: La Haute-Mésopotamie au lle millénaire av. J.-C. París: Collège de France. 
